\title{
NÚMEROS COMPLEXOS: DE GAUSS ÀS APLICAÇÕES NO GEOGEBRA
}

\section{COMPLEX NUMBERS: FROM GAUSS TO GEOGEBRA APPLICATIONS}

\author{
Marta Maria de Azevedo Silva ${ }^{1}$ \\ IFRN - Campus Santa Cruz \\ Jéssica Santos Pereira ${ }^{2}$ \\ IFRN - Campus Santa Cruz \\ Enne Karol Venancio de Sousa ${ }^{3}$ \\ IFRN - Campus Santa Cruz
}

\begin{abstract}
Resumo
Os números complexos passaram por diversas mudanças até chegar a sua representação atual, assim como vários matemáticos contribuíram para sua forma, porém a representação que conhecemos hoje foi a que se popularizou na Matemática. O presente artigo consiste no desenvolvimento de uma pesquisa na disciplina de História da Matemática, do curso de Licenciatura em Matemática do IFRN - Campus Santa Cruz, sobre a contribuição de Carl Friedrich Gauss nos estudos e representação dos números complexos. O objetivo deste artigo é mostrar a representação algébrica e geométrica dos números complexos partindo da utilização da história da Matemática para sua contextualização e do uso de um software, o GeoGebra, para fazer aplicações de sua representação geométrica; unindo o estudo de sua representação através de Gauss e a utilização da tecnologia para essa representação. Fazendo conexão de um assunto teoricamente antigo com os recursos atuais. A metodologia utilizada neste artigo é de cunho qualitativo por ter como campo empírico a utilização de recursos tecnológicos no processo de representação geométrica dos números complexos. Dessa forma, a partir de uma investigação bibliográfica em artigos científicos de domínio público, presentes na rede mundial de computadores, e livros, percebeu-se a eficiência e clareza do GeoGebra na representação geométrica dos complexos. A pesquisa na qual se insere essa comunicação tem como finalidade a formação dos licenciandos para a pesquisa no ensino superior. Diante do que foi exposto no trabalho a partir de artigos, em meios eletrônicos, e livros, nesses foram encontrados referências históricas sobre Gauss e a sua contribuição para forma algébrica dos números complexos através da história da Matemática. Assim, percebeu-se a contribuição da tecnologia no estudo dos números complexos, possibilitando ser feito com que se tornassem grandes aliados e a possibilidade de fazer essa representação, através da adaptação no plano cartesiano, tornando-a significativa.
\end{abstract}

\footnotetext{
${ }^{1}$ marta.maria.11@ hotmail.com

2 jecasp@outlook.com

3enne.sousa@ifrn.edu.br
} 
Palavras-chave: Gauss; GeoGebra; História da Matemática; números complexos; representação geométrica.

\begin{abstract}
The complex numbers underwent several changes until arriving at its present representation, just as several mathematicians contributed to its form, however the representation that we know today was the one that became popular in Mathematics. The present article consists in the development of a research in the discipline of Mathematical History, of the Degree in Mathematics of the IFRN - Campus Santa Cruz, on the contribution of Carl Friedrich Gauss in the studies and representation of the complex numbers. The objective of this article is to show the algebraic and geometric representation of the complex numbers starting from the use of the history of Mathematics for its contextualization and the use of a software, GeoGebra, to make applications of its geometric representation; uniting the study of its representation through Gauss and the use of technology for this representation. Connecting a theoretically old subject with the current resources. The methodology used in this article is qualitative because it has as an empirical field the use of technological resources in the process of geometric representation of complex numbers. Thus, from a bibliographical research in scientific articles of public domain, present in the worldwide network of computers, and books, the efficiency and clarity of GeoGebra in the geometric representation of the complexes was perceived. The research in which this communication is inserted has the purpose of training the graduates for research in higher education. Faced with what has been exposed in the work from articles, in electronic media, and books, in these have been found historical references on Gauss and his contribution to algebraic form of complex numbers through the history of Mathematics. Thus, the contribution of technology in the study of complex numbers was perceived, enabling them to become great allies and the possibility of making this representation through adaptation on the Cartesian plane, making it significant.
\end{abstract}

Keywords: Gauss; GeoGebra; History of Mathematics; complex numbers; geometric representation.

\title{
Introdução
}

Os números complexos passaram por diversas mudanças até chegar a sua representação atual, assim como vários matemáticos contribuíram para sua forma, porém a representação que conhecemos hoje foi a que se popularizou na Matemática.

O presente artigo consiste no desenvolvimento de uma pesquisa na disciplina de História da Matemática, do curso de Licenciatura em Matemática do IFRN - Campus Santa Cruz, sobre a contribuição de Carl Friedrich Gauss nos estudos e representação dos números complexos.

Gauss (1777-1855) desde cedo externou sua enorme capacidade para a Matemática. Conta-se que em uma atividade quando criança, para a soma de todos os números de 1 a 100, ele usou a expressão $n(n+1) / 2$. Aos dezoito anos demonstrou que 
um polígono regular de dezessete lados poderia ser construído apenas com a utilização de régua e compasso. Mas ele só ganhou fama aos vinte e três anos, quando computou a órbita do planetoide de Ceres.

Um tratado publicado por Gauss em 1831 teve grande importância histórica. Segundo Mol (2013):

\begin{abstract}
Um tratado publicado por Gauss em 1831 teve importância histórica por apresentar a representação geométrica dos números complexos, estabelecendo a correspondência entre o número $z=x+i y$ e o ponto do plano cartesiano de coordenadas $(x, y)$. Uma representação geométrica similar dos números complexos já havia sido obtida, em 1797, pelo norueguês Caspar Wessel (1745-1818). No entanto, foi o trabalho de Gauss que a popularizou dentro da comunidade matemática, tanto que o plano dos números complexos é hoje conhecido como plano Gaussiano (MOL, 2013, p. 126).
\end{abstract}

Os números complexos são dados por sua forma algébrica sendo representados por $\mathrm{z}=\mathrm{x}+\mathrm{yi}$ e por sua forma geométrica representada no plano cartesiano fazendo a utilização das coordenadas x e y, dos eixos das abcissas e ordenadas respectivamente. Para a representação geométrica será utilizado o software GeoGebra. O GeoGebra (junção das palavras Geometria e Álgebra) é um aplicativo do ensino da matemática de forma dinâmica que combina conceitos de geometria e álgebra em um único software. O programa permite realizar construções geométricas com a utilização de pontos, retas, segmentos de reta, circunferências, polígonos, etc., assim como permite inserir funções e alterar todos esses objetos dinamicamente.

O objetivo deste artigo é mostrar a representação algébrica e geométrica dos números complexos fazendo a utilização da história da Matemática para sua contextualização até o uso de um software, o GeoGebra, para fazer aplicações de sua representação geométrica. Unindo o estudo de sua representação através de Gauss e a utilização da tecnologia para essa representação. Possibilitando a conexão de um assunto teoricamente antigo com recursos atuais. A padronização do formato a ser utilizado nos artigos é essencial para a correta edição dos anais do evento. Este documento descreve os aspectos da formatação do modelo de artigos, portanto serve como referência. 


\section{Gauss e os Números Complexos}

Gauss pretendeu demonstrar o teorema da reciprocidade biquadrática, porém técnicas utilizadas por ele não possibilitaram uma demonstração geral do teorema, apenas para casos específicos. Segundo Grimberg (2014):

[...] Isso levou Gauss a elaborar uma extensão do domínio dos números inteiros ao dos números imaginários inteiros. $\mathrm{O}$ passo decisivo reside na consideração que um número primo da forma $2 p+1$ é soma de dois quadrados; e assim botem se uma fatoração do número primo como produto de dois números imaginários: $2 p+1=a^{2}+b^{2}=(a+b i)(a-b i)$. Este método já tinha sido descoberto e utilizado por Euler (170, p. 208 e sq.) para encontrar uma fatoração dos números da forma $x^{2}+c y^{2}$. Mas Euler não justificava a possibilidade de tratar os números inteiros imaginários da mesma maneira que os números inteiros (GRIMBERG, 2014, p. 149).

O desenvolvimento dos números complexos se deu de maneira longa, alcançando a contribuição de diversos matemáticos. Euler foi um dos que contribuiu com sua extensão, porém não conseguiu justificar a possibilidade de atenção às raízes negativas como às raízes positivas.

Os números complexos então surgiram da inexistência de soluções para algumas equações quadráticas. Segundo Júnior (2009):

Os números complexos são freqüentemente associados à resolução de equações quadráticas cujas soluções são expressas por raízes quadradas de números negativos. Evoca-se um contexto histórico para justificar a necessidade de se introduzir estes números na matemática, ainda que a sua história não seja detalhada.

[...] As equações quadráticas que apareciam na matemática grega surgiam de investigações geométricas que utilizavam figuras como círculos e parábolas. No entanto, algumas soluções não podiam ser obtidas através destas construções, o que caracterizava a inexistência de solução das equações quadráticas correspondentes (JÚNIOR, 2009, p. 11).

Com isso, os números complexos são extensão da função quadrática para as quais não eram aceitas as raízes quadradas de números reais negativos, adquirindo a forma $\mathrm{z}=\mathrm{x}+\mathrm{yi}$ formulada por Gauss, sendo o matemático que conseguiu justificar essa atenção às raízes negativas, e assim sendo popularizada na Matemática.

\section{Representação Geométrica}

A forma da representação geométrica dos números complexos se deu início em 1673, obtendo-se, assim, o plano de Argand-Gauss. De acordo com Silva (2008): 
A forma de representação geométrica dos Números Complexos, que hoje conhecemos como Plano de Argand-Gauss, teve início em 1673, com John Wallis que publica em 1673 a primeira representação de uma possível localização da solução geométrica de problemas envolvendo raízes quadradas de números reais negativos. Iniciava-se, então, a representação de números complexos por pontos do plano, uma maneira de geometrizar a álgebra dos complexos (SILVA, 2008, p. 15).

A representação geométrica dos números complexos é remota assim como seu estudo, que com seu aprofundamento pode ser realizada de diversas maneiras.

Entre as diversas possibilidades da representação geométrica dos números complexos está a representação utilizando o plano cartesiano, fazendo a aplicação no GeoGebra. Segundo Bastos (2013):

[...] Vemos a ênfase nas "múltiplas possibilidades da representação geométrica de um número complexo $\mathrm{z}$, que tem como imagem um ponto no plano, como um par $(\mathrm{x}, \mathrm{y})$ de números reais, ou escrito na forma $\mathrm{z}=\mathrm{x}+\mathrm{yi}$. Assim, como a reta foi necessária e suficiente para acolher todos os números reais, racionais e irracionais, veremos que, com a expansão do campo numérico para incluir números que possam ser raízes quadradas de negativos, será necessário (e suficiente) todo plano cartesiano, que servirá de inspiração para construção do plano complexo, suporte para representação de todos os números complexos" (BASTOS, 2013, p. 40).

A representação geométrica dos números complexos no plano de Argand-Gauss teve início em 1673 e obteve as várias possibilidades de representação, porém a mais utilizada é sua adaptação no plano cartesiano.

\section{Metodologia}

A metodologia utilizada neste artigo é de cunho qualitativo por ter como campo empírico a utilização de recursos tecnológicos no processo de representação geométrica dos números complexos. Dessa forma, a partir de uma investigação bibliográfica em artigos científicos de domínio público, presentes na rede mundial de computadores, e livros, percebeu-se a eficiência e clareza do GeoGebra na representação geométrica dos complexos. Pretendendo assim dinamizar e diferenciar essa representação. Bastos (2013) descreve como essa representação é feita. Segundo ele:

As coordenadas de um ponto de $\mathbb{R}^{2}$, por exemplo $(\mathrm{x}, \mathrm{y})$, constituem a parte real e a parte imaginária de um número complexo, respectivamente. Portanto, podemos definir: a imagem do complexo $\mathrm{z}=\mathrm{x}+\mathrm{yi}$ é o ponto $(\mathrm{x}, \mathrm{y})$ do plano cartesiano. Podemos, também, chamar o segmento orientado que liga a origem do plano à imagem de $\mathrm{z}$ de vetor representativo desse número complexo; vetor esse, $\overrightarrow{O z}$, com componentes x e y. Neste contexto, 
chamaremos o plano cartesiano de plano complexo o eixo das abcissas de eixo real e o eixo das ordenadas de eixo imaginário (BASTOS, 2013, p. 25).

Com isso, a utilização do software nesse processo de representação geométrica dos números complexos faz com que os estudos desse assunto, que existe há muito tempo, seja feito de forma mais atualizada, no caso, com a utilização da tecnologia e das fontes de pesquisa utilizadas nesse processo.

Assim, a metodologia utilizada no desenvolvimento e aplicação, facilitará o alcance do objetivo principal, possibilitando a representação dos números complexos de maneira tecnológica e diferenciada.

\section{Representação algébrica e geométrica dos números complexos}

Define-se o conjunto dos números complexos, sendo representado por $\mathbb{C}$, o conjunto dos pares ordenados de números reais para os quais estão estabelecidas a igualdade, a adição e a multiplicação.

Adotaremos dois elementos, $(\mathrm{a}, \mathrm{b})$ e $(\mathrm{c}, \mathrm{d})$, de $\mathbb{R}^{2}$ para dar três essenciais definições:

Igualdade: dois pares ordenados são iguais se, e somente se, apresentarem primeiros e segundos, respectivamente, termos iguais.

$$
(a, b)=(c, d) \leftrightarrow a=c \text { e b }=d
$$

Adição: é a soma dos primeiros e a soma dos segundos termos dos pares dados.

$$
(\mathrm{a}, \mathrm{b})+(\mathrm{c}, \mathrm{d})=(\mathrm{a}+\mathrm{c}, \mathrm{b}+\mathrm{d})
$$

Multiplicação: é o produto de dois pares ordenados cujo primeiro termo é a diferença entre o produto dos primeiros termos e o produto dos segundos termos dos pares dados e o segundo termo é a soma dos produtos do primeiro termo de cada par dado pelo segundo termo do outro.

$$
(a, b) .(c, d)=(a c-b d, a d+b c)
$$

Utilizando as definições para fazer as aplicações, temos:

Dados $\mathrm{z}_{1}=(4,1)$ e $\mathrm{z}_{2}=(3,2)$, calcular $\mathrm{z}_{1}+\mathrm{z}_{2}, \mathrm{z}_{1}, \mathrm{z}_{2}$ e $\mathrm{z}_{1}^{2}$.

Calculando:

$Z_{1}+Z_{2}=(4,1)+(3,2)=(4+3,1+2)=(7,3)$

$Z_{1} \cdot Z_{2}=(4,1) .(3,2)=(4 \cdot 3-1 \cdot 2,4 \cdot 2+1 \cdot 3)=(10,11)$

$\mathrm{Z}_{1}^{2}=\mathrm{Z}_{1} \cdot \mathrm{Z}_{1}=(4,1) .(4,1)=(4 \cdot 4-1 \cdot 1,4 \cdot 1+1.4)=(15,8)$ 
Chama-se por unidade imaginária e representada por $i$ o número complexo $(0,1)$. Observamos que:

$\mathrm{I}^{2}=\mathrm{i} . \mathrm{i}=(0,1) \cdot(0,1)=(0.0-1.1,0.1+1.0)=-1$. Por isso a propriedade básica da unidade imaginária é: $\mathrm{i}^{2}=-1$.

Para todo $\mathrm{n} \in \mathrm{N}$, temos:

$I^{4}=1, i^{3}=-i, i^{2}=-1, i=i$.

Define-se conjugado do complexo $\mathrm{z}=\mathrm{x}+$ yi ao complexo $\bar{z}=\mathrm{x}-\mathrm{yi}$, isto é:

$\mathrm{Z}=\mathrm{x}+\mathrm{yi} \leftrightarrow \bar{z}=\mathrm{x}-\mathrm{yi}$

Fazendo uso das definições citadas anteriormente, serão mostradas as representações geométricas das operações feitas com os seguintes números complexos que serão adaptados por pares ordenados.

Dados: $Z_{1}=(1,6)$ e $Z_{2}=(4,7)$, calcular $Z_{1}+Z_{2}, Z_{2}-Z_{1}, Z_{1} . Z_{2}$ e $Z_{1} / Z_{2}$.

$\mathrm{Z}_{1}+\mathrm{Z}_{2}=(1,6)+(4,7)=(1+4,6+7)=(5,13)$

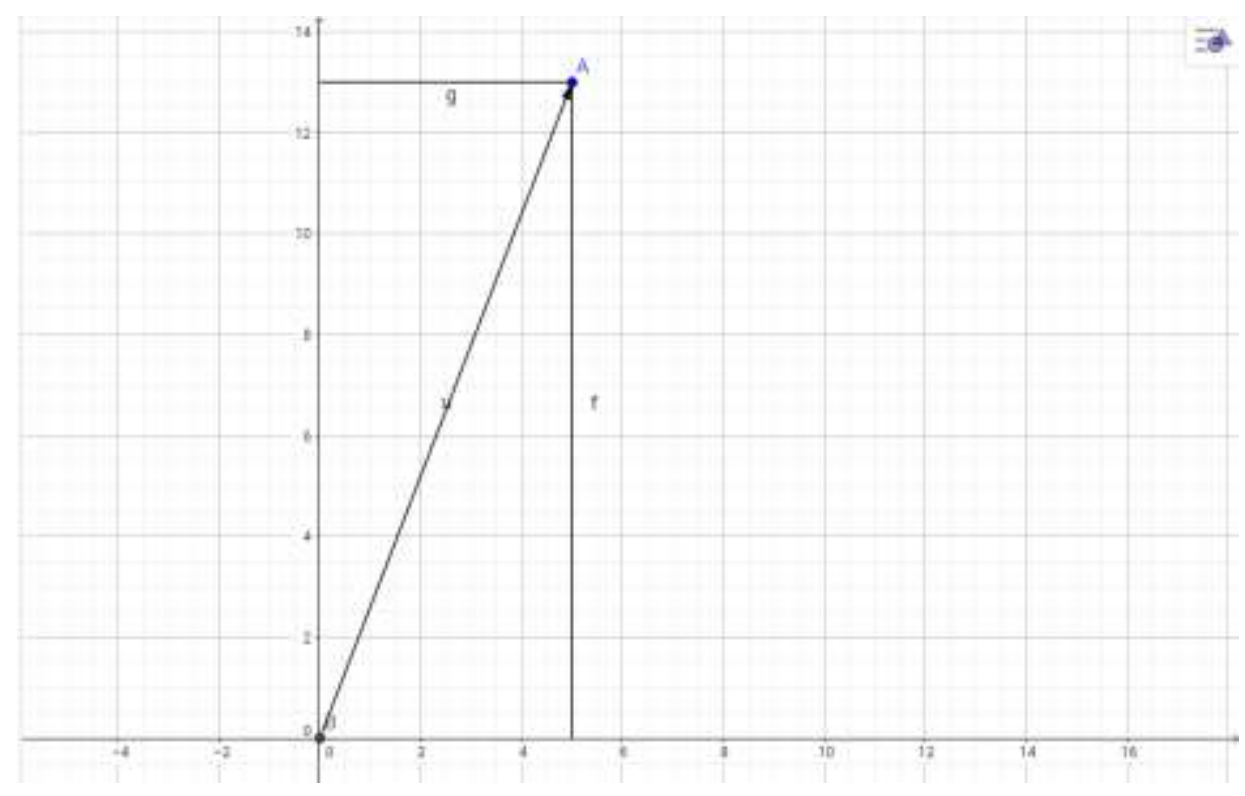

Figura 1: Representação geométrica da adição

Fonte: Acervo das autoras

$\mathrm{Z}_{2}-\mathrm{Z}_{1}=(4,7)-(1,6)=(4-1,7-6)=(3,1)$ 


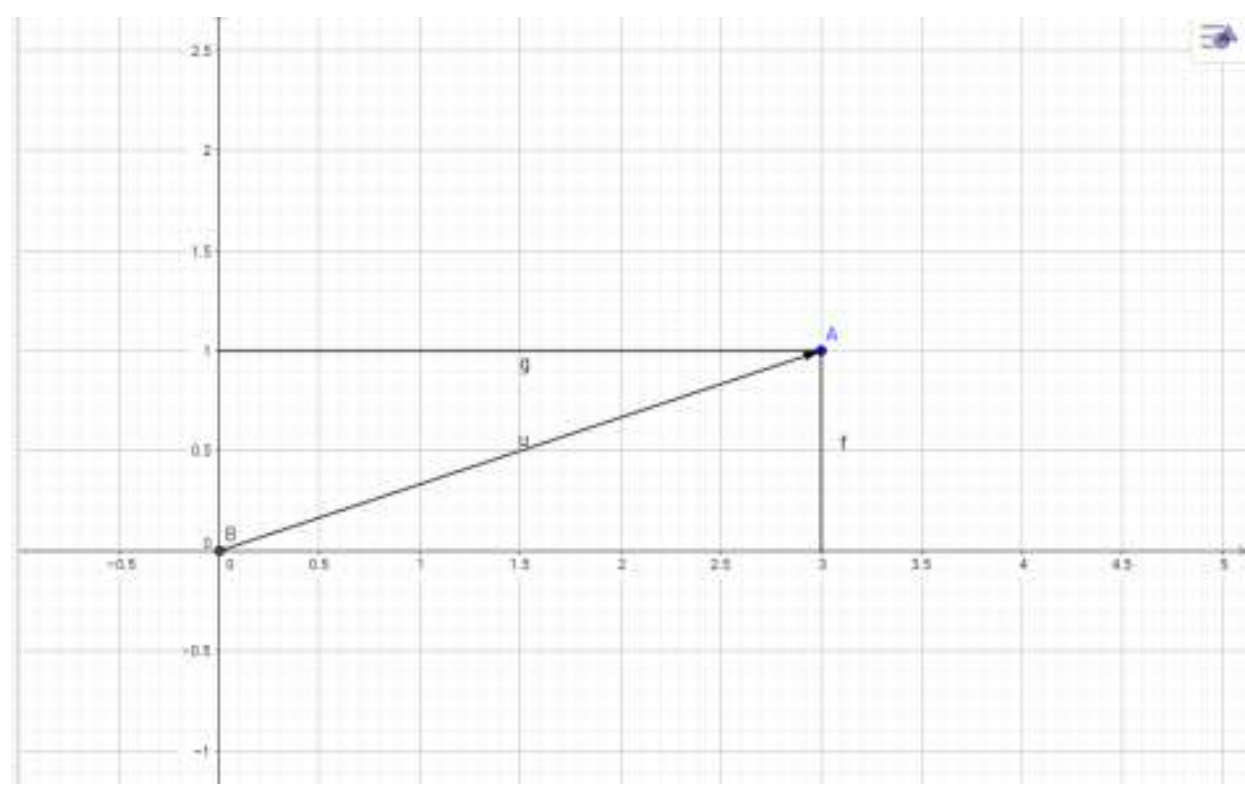

Figura 2: Representação geométrica da subtração

Fonte: Acervo das autoras

$Z_{1} \cdot Z_{2}=(1,6) \cdot(4,7)=(1.4-6.7,1.7+6.4)=(-38,31)$

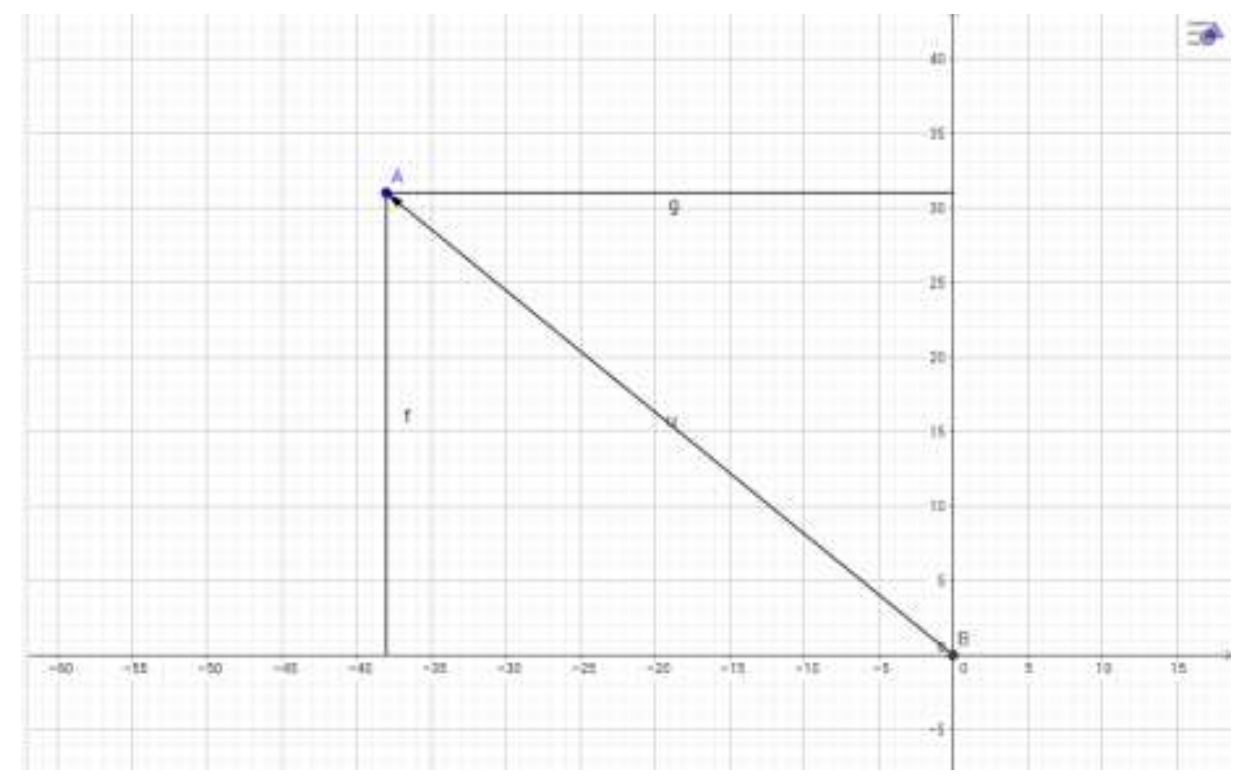

Figura 3: Representação geométrica da multiplicação

Fonte: Acervo das autoras

$\mathrm{Z}_{1} / \mathrm{Z}_{2}=\mathrm{Z}_{1} \cdot \overline{\mathrm{Z}}_{2}=(1,6) \cdot(4,-7)=(1.4-6 .(-7), 1 .(-7)+6.4)=(46,17)$ 


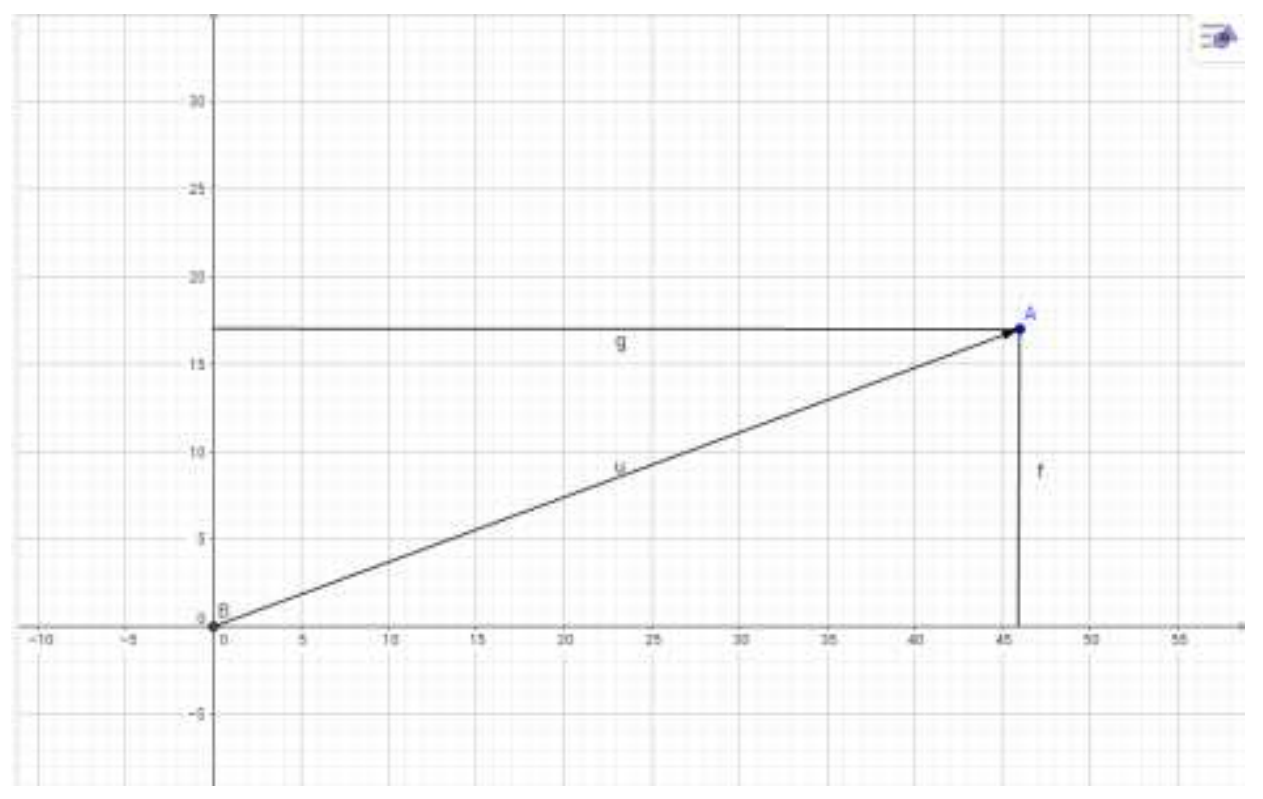

Figura 4: Representação geométrica da divisão Fonte: Acervo das autoras

\section{Considerações Finais}

A partir do objetivo deste artigo que foi mostrar a representação algébrica e geométrica dos números complexos fazendo a utilização da história da Matemática para sua contextualização até o uso de um software, o GeoGebra, para fazer aplicações de sua representação geométrica, e da metodologia utilizada, de cunho qualitativo por ter como campo empírico a utilização de recursos tecnológicos no processo de representação geométrica dos números complexos, desde uma investigação bibliográfica em artigos científicos de domínio público, presentes na rede mundial de computadores, e livros, percebeu-se a eficiência e clareza do GeoGebra na representação geométrica dos complexos.

Com isso, foram feitas algumas aplicações de operações com os números complexos sendo utilizado o software GeoGebra, com o plano cartesiano na forma de par ordenado $(\mathrm{x}, \mathrm{y})$, com a coordenada abscissa para representação dos números reais e a coordenada ordenada para representação dos números imaginários.

Assim, percebeu-se a contribuição da tecnologia no estudo dos números complexos, possibilitando essa aliança e a possibilidade de fazer essa representação, através da adaptação no plano cartesiano, tornando-a significativa. 


\section{Referências}

BASTOS, Leonardo de Mattos. NÚMEROS COMPLEXOS E GEOGEBRA. Rio Claro, 2013. 57 p. Disponível em: <

https://repositorio.unesp.br/bitstream/handle/11449/92411/bastos_lm_me_rcla.pdf?sequ ence $=1>$. Acesso em: dez. 2017.

GRIMBERG, Gerard Emile. GAUSS, OS RESÍDUOS BIQUADRÁTICOS E A REPRESENTAÇÃO GEOMÉTRICA DOS NÚMEROS COMPLEXOS. Revista Brasileira de História da Matemática - Vol. 14 n 29 - p. 145-166. 2014. Disponível em: <http://www.rbhm.org.br/issues/RBHM\%20-\%20vol.14,no29/7\%20\%20Grimberg.pdf $>$. Acesso em: dez. 2017.

JÚNIOR, Ulício Pinto. A HISTÓRIA DOS NÚMEROS COMPLEXOS: “DAS QUANTIDADES SOFISTICADAS DE CARDANO ÀS LINHAS ORIENTADAS DE ARGAND”. Rio de Janeiro, 2009. Disponível em: < http://www.pg.im.ufrj.br/pemat/12\%20Ulicio\%20Pinto.pdf >. Acesso em: dez. 2017.

MOL, Rogério Santos. Introdução à História da Matemática. Belo Horizonte: CAED - UFMG, 2013.

SILVA, Edvaldo Lima da. Números e funções complexas: representação e interpretação gráfica / Edvaldo Lima da Silva, Aguinaldo Robinson de Souza [e] Emília de Mendonça Rosa Marques. - São Paulo: Cultura Acadêmica: Universidade Estadual Paulista, Pró-Reitoria de Graduação, 2008, 73 p. Disponível em: < http://wwwp.fc.unesp.br/ edvaldo/livro/preview.html >. Acesso em: dez, 2017. 